\title{
Tumor del estroma gastrointestinal (TEGI): terapia adyuvante ¿Cuánto tiempo?
}

\section{For How Long Should Adjuvant Therapy for Gastrointestinal Stromal Tumors (GIST) Be Administered?}

Ricardo Oliveros Wilches, MD, ${ }^{1}$ Ana D. Bonilla Castañeda, MD, ${ }^{1}$ Helena Facundo Navia, MD, ${ }^{1}$ Ricardo Sánchez Pedraza, MD. ${ }^{2}$

1 Grupo de Cirugía Gastrointestinal y Endoscopia Digestiva, Instituto Nacional de Cancerología. Bogotá, Colombia.

2 Grupo de Investigación Clínica, Instituto Nacional de Cancerología. Facultad de Medicina, Universidad Nacional de Colombia. Bogotá, Colombia.

Fecha recibido: $29-02-16$

Fecha aceptado: $01-11-16$

\begin{abstract}
Resumen
La cirugía es el tratamiento establecido para pacientes con tumor del estroma gastrointestinal (TEGI) primario completamente resecable. Luego de la cirugía, se pueden presentar recurrencias hasta en $50 \%$ de los casos en los 2 primeros años, especialmente en pacientes con alto riesgo. Esto justifica la terapia adyuvante con imatinib (IMB). Debido a la evidencia clínica, se sabe que 3 años de tratamiento es el tiempo establecido para pacientes con alto riesgo de recurrencia; sin embargo, también se conoce por los mismos estudios clínicos, que las recurrencias comienzan a detectarse de nuevo 6 a 9 meses después de suspender el medicamento.

Se presentaron 3 pacientes llevados a cirugía con resección completa de la lesión, quienes recibieron imatinib por 3 años. Meses después de suspender el medicamento, presentaron recurrencia, lo que obligó nuevamente su administración. La duración óptima del tratamiento adyuvante con IMB no está establecida, y no es claro si el IMB realmente cura la enfermedad. Por este motivo, se plantea la necesidad de revisar por cuánto tiempo se debe administrar la terapia adyuvante con IMB y el efecto que el IMB realmente tiene sobre la enfermedad. En conclusión, por la evidencia actual se sabe que la recomendación es de 3 años de tratamiento con IMB como terapia adyuvante, pero con base en la experiencia diaria y con las recomendaciones de expertos, existen pacientes que probablemente necesiten continuar con imatinib por mucho más tiempo mientras se informan los resultados de los estudios clínicos de 5 años de tratamiento con IMB.
\end{abstract}

\section{Palabras clave}

Tumor estromal gastrointestinal, terapia adyuvante, imatinib, tratamiento, TEGI metastásico y/o avanzado, estratificación de riesgo, perfil molecular, estado mutacional.

\section{Abstract}

Surgery is the established treatment for patients with primary gastrointestinal stromal tumors (GIST) that are completely resectable. After surgery, up to $50 \%$ of patients suffer recurrences in the first two years. This is especially true for high risk patients. This is the justification for adjuvant therapy with imatinib (IMB). Based on clinical evidence, three years is the established treatment time for patients at high risk of recurrence. However, the same clinical studies also show that recurrences begin to be detectable six to nine months after discontinuation of the drug. We present the cases of three patients who underwent complete resection of GIST who then received imatinib for three years. Months after the medication was discontinued, recurrences required administration of the drug to be restarted. The optimal duration of adjuvant therapy with IMB has not been established, and it is unclear whether IMB actually cures the disease. For this reason, a review of how long adjuvant therapy with IMB should be administered and of what the effect of IMB on the disease really is was needed. In conclusion, on the basis of currently available evidence, we know that the recommendation is three years of treatment with IMB as adjuvant therapy, but, based on daily experience and expert recommendations, there are patients who probably need to continue treatment with imatinib for much longer while we wait for reports of the results of clinical studies of five year IMB treatment.

\section{Keywords}

Gastrointestinal stromal tumor, adjuvant therapy, imatinib, treatment, metastatic and/or advanced GIST, risk stratification, molecular profile, mutational state. 


\section{INTRODUCCIÓN}

Los tumores del estroma gastrointestinal (TEGI) son los tumores mesénquimales más frecuentes del tracto gastrointestinal, con una incidencia estimada de 10 a 20 casos por 1 millón de habitantes $(1,2)$. El sitio donde se presenta con mayor frecuencia es el estómago, seguido por el intestino delgado, colon, recto, esófago, también se describen en el mesenterio y retroperitoneo. Los TEGI se originan de las células intersticiales de Cajal, que son las mismas células del marcapaso intestinal; ambas expresan la proteína KIT y tienen características ultraestructurales similares $(2,3)$.

Varios estudios muestran que la activación constitutiva del receptor c-KIT desempeña un papel central en la patogénesis de la mayoría de los TEGI, esto se produce como consecuencia de mutaciones puntuales que pueden ocurrir tanto en la región extracelular, como en la intracitoplasmática del receptor tirosina quinasa $(3,4)$.

El manejo principal de los TEGI es quirúrgico con resección completa de la lesión; sin embargo, $30 \%$ a $50 \%$ de los pacientes llevado a resección completa pueden presentar recurrencia, especialmente en los primeros 2 años. La sobrevida a los 5 años de los pacientes con TEGI completamente resecados es de 50\% (5). Estos datos sirven como base de la necesidad de la terapia adyuvante.

Antes de la era del IMB, los pacientes con lesiones tipo TEGI resecables, se llevaban a cirugía y luego se manejaban bajo observación, ya que ni la quimioterapia ni la radioterapia tienen papel en el manejo de esta patología $(5,6)$. Desde el año 2002, el IMB se aprobó para el manejo del TEGI metastásico; un tiempo después se utilizó y aprobó por 12 meses como manejo adyuvante (7). No obstante, una vez se suspendía el medicamento, los pacientes presentaban recurrencia de la enfermedad en una proporción importante, lo que obligaba a la nueva administración del IMB, afortunadamente con buena respuesta (8). Posteriormente, se demostró que 36 meses de tratamiento con IMB era mejor que 12 meses; sin embargo, una vez más se observó que si se suspendía el medicamento, se presentaba recurrencia $(9,10)$.

Se sabe que el manejo estándar en el cuidado de los pacientes con TEGI con alto riesgo de recurrencia es la terapia adyuvante sistémica con IMB, pero la duración óptima del tratamiento es objeto de debates (11).

\section{PATOLOGÍA MOLECULAR}

Los TEGI se originan de las células intersticiales de Cajal. El gen c-KIT, localizado en el cromosoma 4q11-21, codifica un receptor transmembrana de tipo III tirosina quinasa (KIT), ligando el factor de crecimiento conocido como factor de las células madre. La unión de este ligando al receptor de membrana, determina la dimerización que sigue con la activación de la tirosina quinasa, localizada en la región intracelular; ello a su vez, determina la activación de la cascada intracelular que conduce al control de la diferenciación, proliferación y crecimiento celular (figura 1) $(3,12)$.

$85 \%$ de los TEGI tiene mutaciones activadas en el c-KIT, $5 \%$ de las mutaciones en el factor de crecimiento plaquetario (PDGFRA) y el resto no contiene mutaciones identificadas, que se conocen como tipo salvaje. Las mutaciones en el exón 11 del c-KIT son las más comunes y tienen un alto grado de respuesta al tratamiento inicial con imatinib. Las mutaciones del exón 9 c-KIT son las segundas mutaciones más comunes en este receptor transmembrana, y se asocian con una sensibilidad o respuesta diferente al IMB $\mathrm{y}$ a otros inhibidores de tirosina quinasa (ITK). Las mutaciones en los exones 13 y 17 del c-KIT se describen como menos comunes, y en algunos casos se relacionan con resistencia primaria o secundaria al $\operatorname{IMB}(11,13)$.

Las mutaciones en los exones 12, 14 y 18 del PDGFRA se encontraron y se consideran como la causa de varios TEGI que no tienen mutaciones activadas en el c-KIT. Presentan su propio pronóstico basado en la respuesta a los medicamentos actualmente disponibles. Los TEGI tipo salvaje sin mutaciones en el c-KIT o en el PDGFRA, poseen una expresión aumentada del factor 1 de crecimiento, como la insulina. Potencialmente, muestran un peor pronóstico y se asocian con un mayor índice mitótico, tamaño y cifras de recurrencia y de metástasis $(13,14)$.

El desarrollo de las terapias blanco revolucionó el tratamiento del TEGI. Sin embargo, el análisis del tumor individual (perfil molecular) es el primer paso para maximizar el éxito del tratamiento, ya que permite identificar a los pacientes que se benefician de tales terapias $(13,15)$.

$\mathrm{El}$ análisis mutacional puede proporcionar la información de no tratamiento, como es el caso de las mutaciones PDGFRA D842V, que son insensibles a IMB, o puede sugerir el incremento de la dosis de IMB, como es el caso de TEGI con mutación del exón 9. Sin embargo, a pesar del beneficio de la información del estado mutacional, no existe una aproximación terapéutica establecida para cada subtipo molecular de TEGI. Las guías de la mayoría de los países europeos, recomiendan el análisis mutacional, en contraste con EE. UU. donde menos del 10\% de los pacientes con TEGI tiene perfil molecular. El análisis mutacional debe ser tan común como en otros tipos de tumores, por ejemplo en el de mama (13). El estado mutacional se recomienda en todos los pacientes con TEGI en quienes se plantea la terapia con ITK (16).

\section{ESTRATIFICACIÓN DE RIESGO}

Varios factores de riesgo se asocian con la recurrencia tumoral, por ello, la estratificación del riesgo es esencial 


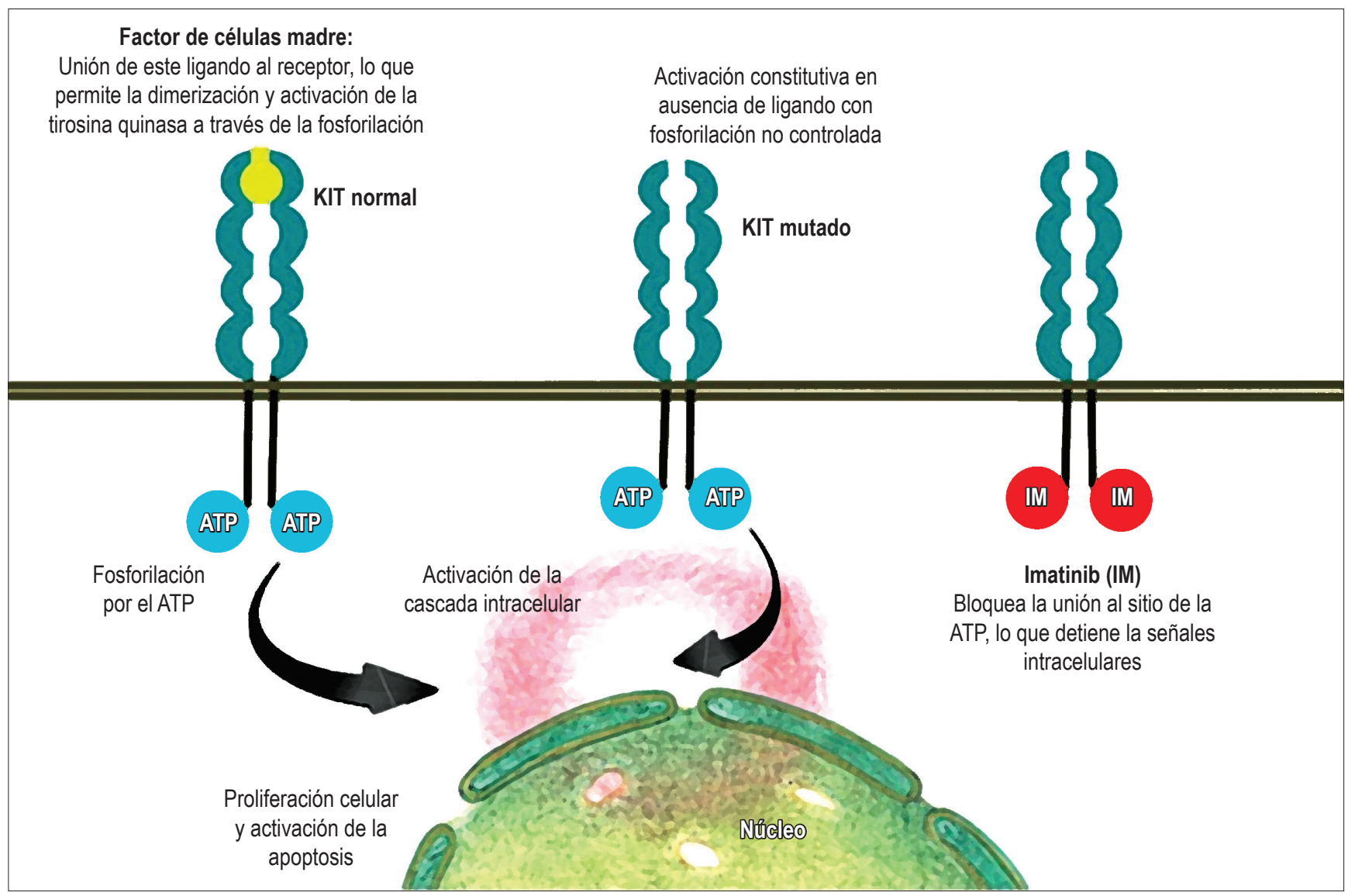

Figura 1. Gen KIT normal, KIT mutado y acción de imatinib.

para identificar los pacientes con TEGI que se pudieran beneficiar de la terapia adyuvante. Estos factores de riesgo son el índice mitótico, la localización del tumor, el tamaño y la ruptura durante la manipulación quirúrgica $(11,17)$.

La resección quirúrgica completa es posible aproximadamente en $85 \%$ de los casos, y es de importancia en la sobrevida media de los pacientes con TEGI. Cuando se realiza una resección completa, la sobrevida es de 66 meses comparada con 22 meses cuando es incompleta $(11,17)$.

El primer esquema para la determinación del riesgo biológico fue el producto del consenso llevado a cabo por el National Institute of Health (NIH) en 2001, en donde se tuvo como factores de riesgo el tamaño tumoral y el índice mitótico. Luego, este esquema se modificó teniendo en cuenta el trabajo de Miettinen y colaboradores del grupo de Patología del Instituto de las Fuerzas Armadas (AFIP). Este sistema de clasificación incluyó la localización del tumor dentro del esquema de pronóstico. La ruptura del tumor y la ubicación no gástrica se plantearon más adelante como variables pronosticas independientes en los sistemas de clasificación de riesgo (18).
En el sistema de clasificación modificado, pacientes con TEGI no gástricos y tamaño menores o iguales de $5 \mathrm{~cm}$, con índice mitótico $>5 \times 50$ campos de alto poder (CAP); TEGI no gástricos con tamaño de 5 a $10 \mathrm{~cm}$ y $<5$ mitosis por $50 \mathrm{CAP}$ o ruptura del tumor, se relacionaron con riesgo de recurrencia mayor de 50\% (11).

Miettinen y colaboradores observaron que pacientes con TEGI gástricos mayores de $10 \mathrm{~cm}$, con índice mitótico menor de 5x50 CAP, tuvieron una recurrencia de solo $10 \%$. Pero los pacientes con un tumor similar del intestino delgado o del recto, presentaron una recurrencia del más del $50 \%$. Esta clasificación permitió identificar pacientes de riesgo bajo, intermedio y alto, y que estos últimos probablemente se beneficiaran de la terapia adyuvante (18).

Luego de la cirugía, las recurrencias a los 5 años puede ser de 50\%, pero en tumores de gran tamaño puede ser tan alta como de $85 \%$ a $90 \%$ (19). En 2009, Gold implementó un nomograma para la estratificación del riesgo de recurrencia. Recientemente, Rossi y colaboradores desarrollaron un nomograma para predecir la sobrevida global. Estos sistemas de estratificación son el inicio de la investigación 
clínica para medir la eficacia y seguridad de la terapia adyuvante en pacientes con alto riesgo de recurrencia $(20,21)$. Se considera que para mejorar el tratamiento de los TEGI se necesita adicionar el análisis molecular al riesgo de estratificación (22).

\section{TERAPIA ADYUVANTE}

La cirugía permanece como la terapia potencialmente curativa para el TEGI primario. Las guías de la National Comprehensive Cancer NetWork (NCCN) recomiendan la resección quirúrgica de todas las lesiones mayores de 2 cm; sin embargo, los resultados después de la cirugía son todavía pobres debido al riesgo de recurrencia (10).

El objetivo del manejo para pacientes con TEGI primario, es la prevención de la recurrencia y/o de la enfermedad metastásica. La sobrevida a los 5 años para pacientes con TEGI resecados es aproximadamente del 50\% y la mediana para las recurrencias está cerca de los 2 años. Estas estadísticas son el soporte para el uso racional de la terapia adyuvante luego de la cirugía para reducir el riesgo de recurrencia y mejorar el curso clínico y la evolución de estos pacientes (23).

El uso del IMB como terapia adyuvante se apoya en 2 estudios elaborados por el Grupo del Colegio Americano de Cirujanos Oncólogos (ACOSOG) (23). En el primer estudio Z9000, el cual fue un estudio multicéntrico de un solo brazo, 107 pacientes con alto riesgo de recurrencia recibieron $400 \mathrm{mg}$ de IMB por 1 año. El tratamiento con IMB demostró una mejora significativa en la sobrevida libre de recurrencia (SLR) y también mejoría en la sobrevida global comparada con los controles históricos en un seguimiento de 4 años. La SLR al primer, segundo y tercer año fue de $94 \%, 73 \%$ y $61 \%$, respectivamente; mientras que la sobrevida global fue de $99 \%, 97 \%$ y $97 \%$, respectivamente. El IMB adyuvante se toleró bien y $83 \%$ de los pacientes completaron el tratamiento. La mayoría de los eventos adversos fueron de grado de gravedad 1 o 2 (24).

En el segundo estudio Z9001 de la ACOSOG, el cual fue un estudio fase II, aleatorizado y doble ciego, se trataron 713 pacientes a quienes se les había resecado lesiones de $3 \mathrm{~cm}$ o mayores. Los pacientes se aleatorizaron y recibieron $400 \mathrm{mg}$ de IMB al día o placebo por 1 año. DeMatteo y colaboradores, demostraron que $400 \mathrm{mg}$ de IMB al día por vía oral por 1 año, mejoraba la sobrevida libre de recurrencia comparado con placebo ( $98 \%$ en comparación con $83 \%$. HR 0,35; IC 95\%: 0,22-0,53; $p=<0,001)$. No hubo mejoría estadísticamente significativa en la sobrevida global con el IMB (HR 0,66; IC 95\%: 0,22-2,03; $p=, 47$ ). El estudio se suspendió de forma temprana por análisis de eficacia interina y todos los pacientes que recibían placebo pasaron a recibir tratamiento con IMB (25).
Con base en estos resultados, la FDA aprobó el uso de IMB en EE. UU. en el 2008 para el tratamiento adyuvante de los TEGI completamente resecados mayores de $3 \mathrm{~cm}$, sin la guía definitiva sobre la duración óptima del tratamiento ni sobre que pacientes tienen más probabilidad de beneficiarse (23).

Estos estudios apoyaron la evidencia de la recomendación de prolongar la terapia adyuvante. Durante el primer año de tratamiento, solo hubo una recurrencia en el brazo con IMB, comparado con 41 en el brazo de placebo; sin embargo, 6 a 12 meses después de suspender el medicamento, las cifras de recurrencia en el brazo del IMB se incrementaron rápidamente. Esto planteo la pregunta de si la terapia con IMB más allá de 1 año podía extender la sobrevida libre de recurrencia (23).

El trabajo del Grupo Sarcoma Scandinavian (SSG) de Joensuu y colaboradores, comparó la terapia con IMB de 12 meses frente a 36 meses ( $400 \mathrm{mg}$ /día vía oral en 400 pacientes con TEGI de alto riesgo de recurrencia). La mediana de seguimiento fue de 54 meses. Se encontró que 3 años de tratamiento con IMB se relacionaba con mejoría en la SLR y sobrevida global comparado con 1 año de tratamiento. En relación con la sobrevida libre de recurrencia (SLR) a los 5 años los datos fueron $66 \%$ comparado con $48 \%$ con una HR de 0,46 (IC 95\%: 0,32-0,65) y la sobrevida global fue de $92 \%$ en comparación con $82 \%$. Los eventos adversos fueron más frecuentes en el grupo de tratamiento prolongado; sin embargo, la mayoría de los efectos fueron grado $1 \mathrm{o} 2$. Estos datos establecieron un mínimo de 36 meses de IMB adyuvante como un nuevo estándar para los pacientes con TEGI de alto riesgo. A pesar de esto, persisten dudas respecto a si debe continuarse el tratamiento durante más de 3 años. En ambos grupos, luego de 6 a 12 meses de interrupción del IMB adyuvante, las tasas de recurrencia se incrementaron de manera similar. Este hallazgo plantea la cuestión de si las recurrencias se previenen verdaderamente o simplemente se retrasan (9).

Como se describió previamente, la duración óptima de la terapia con IMB todavía necesita definirse. Actualmente, para pacientes con alto riesgo de recaída se desconoce si la duración de la terapia debe ser superior de 3 años y si tales pacientes se deben mantener con IMB adyuvante de forma continua, algo similar a lo que ocurre en los pacientes con leucemia mieloide crónica (LMC) (23).

En pacientes con lesiones marginales o limítrofes donde la cirugía puede implicar resección de órganos vecinos, la terapia neoadyuvante se recomienda porque disminuye la morbilidad quirúrgica por reducción del tamaño y vascularización de la lesión. La terapia adyuvante se indica por lo menos 3 años después de la resección del tumor primario en pacientes que recibieron terapia neoadyuvante. Por esto, la asociación entre terapia neoadyuvante y adyuvante no es sorprendente (16). 
Las guías de práctica clínica de la National Comprehensive Cancer NetWork (NCCN) sugieren IMB adyuvante durante 36 meses en los pacientes con TEGI de alto riesgo (tumor $>5 \mathrm{~cm}$ con alto índice mitótico $>5$ mitosis $\mathrm{x} 50$ $\mathrm{CAP}$ ) o un riesgo de recurrencia $>50 \%$ (19).

El riesgo de recurrencia del TEGI es mayor durante el primer o segundo año luego de suspender la terapia adyuvante. Restablecer la terapia durante este período mitiga este riesgo. Mientras la duración óptima de la terapia adyuvante permanece desconocida, 3 años es mejor que 1 en términos de sobrevida libre de recurrencia y global. Por ello, varios autores recomiendan la terapia adyuvante con IMB durante 3 años como el manejo estándar en los pacientes con TEGI resecados de alto riesgo (19).

Se identificaron 3 medicamentos con beneficio clínico en el TEGI: imatinib, sunitinib y regorafenid (26).

El IMB demostró ser efectivo hasta 8 años sin evidencia de recurrencia $(9,26)$. La eficacia del IMB varía entre los casos y depende de la sensibilidad del tipo de mutación. La estimación del riesgo es de suma importancia cuando se selecciona el paciente que podría beneficiarse de la terapia con IMB (11).

Le Cesne informó los resultados de un estudio aleatorizado de pacientes con TEGI quienes mantuvieron un control de su enfermedad metastásica con 3 años de tratamiento con IMB. Los pacientes se aleatorizaron en grupos que continuaban o interrumpían el tratamiento. El tiempo medio de progresión fue de 9 meses después de la asignación al grupo sin tratamiento. Luego de un seguimiento de 35 meses después de la aleatorización, la SLR a los 2 años fue de $80 \%$ en el grupo que continuaba con el medicamento comparado con $16 \%$ en el grupo que lo interrumpió. Afortunadamente, la reintroducción del IMB se asoció con $100 \%$ de control del tumor en el grupo que se interrumpió. Este trabajo puede asimilarse al manejo adyuvante sabiendo que se trataba de pacientes con enfermedad metastásica (27).

Actualmente, se encuentra en curso un estudio fase II de un solo brazo, no aleatorizado, sobre la evaluación de sobrevida libre de recurrencia luego de resección completa (PERSIST-5) con IMB adyuvante evaluado por 5 años (400 mg vía oral por día); incluye pacientes con TEGI de alto riesgo completamente resecados. El objetivo principal del estudio es evaluar la sobrevida libre de recurrencia. Los informes preliminares de este estudio en desarrollo sugieren un beneficio de la terapia extendida con IMB. Después de un seguimiento de 44 meses, se observó una mejoría significativa en la SLR en pacientes que están tomando IMB. Las cifras de SLR para quienes reciben IMB (35 pacientes) comparado con el seguimiento (55 pacientes), fueron 100\% en comparación con $70,9 \%$ para el primer año; $88 \%$ comparado con $37,8 \%$ para el segundo año y $88 \%$ en comparación con $27,5 \%$ para el tercer año, respectivamente (HR: 0,122; IC 95\%: 0,041-0,363; $p=0,000)$. Aún no se informan sobre los datos de 5 años o más; sin embargo, esta información soporta la evidencia de que la terapia extendida con IMB al menos 3 años, prolonga la SLR de pacientes con alto riesgo de recurrencia (28).

Uno de los objetivos del estudio BFR14 fase III del Grupo Francés de Sarcomas, fue investigar el efecto de continuar comparado con interrumpir la terapia adyuvante con IMB. Este trabajo se realizó en pacientes con TEGI avanzado, mediante la interrupción de la terapia después de 1, 3 o 5 años de tratamiento diario con IMB a $400 \mathrm{mg}$ vía oral. Se encontró que el riesgo de progresión se incrementó con la suspensión del IMB, y los pacientes con progresión de la enfermedad, luego de suspender el medicamento, no lograban siempre el mismo grado de control del tumor que tenían antes de la interrupción de la terapia (29). Los autores del estudio BRF14 concluyen que 5 años de IMB no es suficiente en pacientes con TEGI avanzado para lograr la remisión completa, y se preguntan si estos hallazgos podrían ser similares para el uso de la terapia adyuvante $(30,31)$.

Ahora, se presentarán 3 pacientes con TEGI de alto riesgo manejados con cirugía e IMB como terapia adyuvante, a quienes se les suspendió el medicamento luego de 3 años de administración, con recaída en los meses posteriores a la interrupción y mejoría en el control de su enfermedad con la reiniciación del medicamento. Esto plantea la pregunta de si la terapia con IMB en pacientes con TEGI con alto riesgo de recurrencia y respondedores debe continuar más allá de los 36 meses y por cuánto tiempo más.

\section{Caso 1}

Mujer de 49 años con TEGI gástrico de alto riesgo de mutación del exón 11, se manejó con gastrectomía total, esplenectomía, pancreatectomía distal y colectomía parcial. Recibió IMB desde septiembre del 2010 hasta noviembre del 2013. En enero del 2015, se detectó metástasis hepáticas y se reinició IMB en abril del 2015, con respuesta parcial en su último control de diciembre del 2015 (tabla 1).

\section{Caso 2}

Mujer de 64 años con TEGI gástrico $(>10 \mathrm{~cm})$ manejada con IMB neoadyuvante por 7 meses. Luego de una respuesta importante por reducción del tamaño de la lesión, se le practicó una gastrectomía subtotal del $80 \%$. Consecutivamente, recibió IMB desde febrero del 2012 hasta febrero del 2015. Después de 6 meses, se detectó recurrencia. Se formuló sunitinib, la cual no toleró, por lo que se inicia IMB con adecuada tolerancia y reducción en 
Tabla 1. Características clínicas de pacientes manejados con terapia adyuvante por 3 años con tiempo de recurrencia luego de suspender imatinib

\begin{tabular}{ccccccccc}
\hline Sexo & Edad & Localización & Tamaño & $\begin{array}{c}\text { Índice } \\
\text { mitótico }\end{array}$ & Mutación & Cirugía & $\begin{array}{c}\text { Tratamiento } \\
\text { adyuvante }\end{array}$ & $\begin{array}{c}\text { Tiempo de } \\
\text { recurrencia }\end{array}$ \\
\hline $\mathrm{F}$ & 49 & Gástrico & $20 \times 20 \mathrm{~cm}$ & $>5 \times 50 \mathrm{CAP}$ & Exón 11 & Gastrectomía total & 36 meses & 15 meses \\
$\mathrm{F}$ & 64 & Gástrico & $8 \times 5 \mathrm{~cm}$ & $3 \times 50 \mathrm{CAP}$ & - & Cuña gástrica & 36 meses & 6 meses \\
$\mathrm{M}$ & 62 & Gástrico & $27 \times 25 \mathrm{~cm}$ & $3 \times 50 \mathrm{CAP}$ & Exón 11 & Cuña gástrica & 36 meses & 6 meses \\
\hline
\end{tabular}

el tamaño de la recurrencia en su último control de diciembre de 2015 (figura 2).

\section{Caso 3}

Hombre de 62 años con TEGI gástrico y mutación del exón 11, manejado extrainstitucionalmente con cuña gástrica y luego imatinib por 3 años, con recaída posterior a los 6 meses de suspender el medicamento. Recibió nilotinib por 8 meses con reducción importante del tamaño de la lesión, por lo que en noviembre del 2014, se llevó nuevamente a cirugía con esplenectomía, resección de $10 \mathrm{~cm}$ de intestino delgado, resección parcial del diafragma izquierdo y cuña gástrica. Desde entonces recibió imatinib con adecuado control clínico y sin recurrencia hasta su ultimo control diciembre del 2015 (tabla 1).

\section{DISCUSIÓN}

La duración óptima de la terapia adyuvante con IMB todavía no se conoce. La cirugía es el tratamiento primario para los TEGI resecables, pero $30 \%$ a $50 \%$ de los pacientes puede presentar recurrencias en los primeros 5 años, mientras que aquellos con alto riesgo lo hacen de una forma más temprana. La sobrevida de los pacientes con TEGI metastásico se incrementó hasta 3 veces desde la introducción de los inhibidores de tirosina quinasa $(17,32)$.

La selección de los pacientes para la terapia adyuvante se debe basar en cualquiera de los esquemas de estratificación. Los médicos deben analizar el riesgo de recurrencia del paciente dependiendo de varios factores como el tamaño de la lesión, localización, índice mitótico y ruptura del tumor durante la cirugía. El análisis mutacional es importante a la hora de decidir el tratamiento adyuvante, pero no se toma en cuenta por ningún sistema de estratificación de riesgo $(17,32)$.

Los 3 pacientes documentados tenían lesiones mayores de $10 \mathrm{~cm}(20 \times 20 \mathrm{~cm}, 27 \times 25 \mathrm{~cm}$ y >10 cm). Uno de ellos recibe neoadyuvante con IMB con reducción importante $(8 \times 5 \mathrm{~cm})$ al momento de la cirugía.

El primer paciente referenciado se hubiera beneficiado de este tipo de tratamiento neoadyuvante para disminuir el tamaño y vascularización de la lesión y evitar la necesidad de realizar resección de órganos por contigüidad. Por ello, se plantea si pacientes con lesiones mayores de $10 \mathrm{~cm}$, con alto índice mitótico deban recibir este tipo de manejo; es decir, primero neoadyuvante y, luego de la cirugía, continuar con el IMB por 3 años.
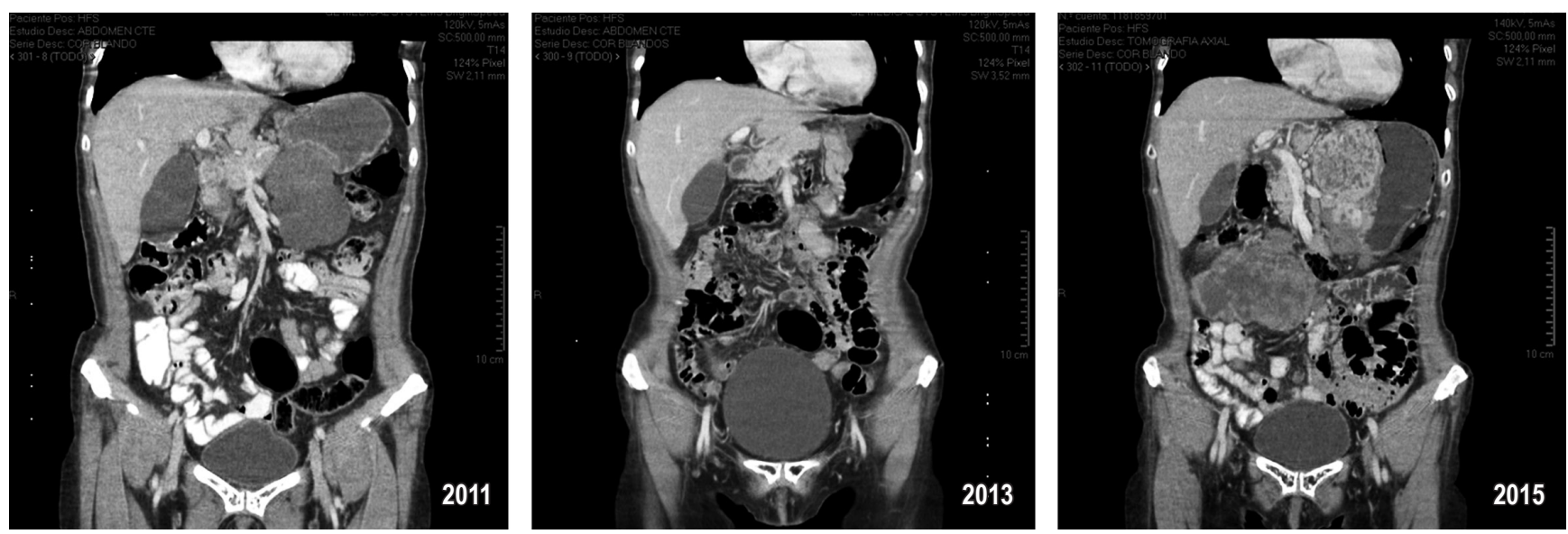

Figura 2. Tomografías del caso 2. Año 2011: inicio; año 2013: en tratamiento; y año 2015: con recurrencia. 
Igualmente, 2 de los pacientes referenciados tenían mutación del exón 11, lo que demuestra la buena respuesta al IMB. En el primer caso, luego de detectarse recurrencia, el reinicio del medicamento mostró respuesta parcial de las lesiones; mientras que en el tercer caso, luego de lograrse la resección quirúrgica completa (R0), el medicamento administrado mostró excelente control sin nueva recurrencia.

Algunos pacientes con alto riesgo de recurrencia, a pesar de cirugías completas macroscópicamente (R0), podrían beneficiarse de la terapia adyuvante con IMB por períodos mayores de 3 años. Esto permanece como una hipótesis con poca evidencia de soporte, sumado al riesgo derivado de la ingesta del medicamento cuando podría ser posible no necesitarlo. Por esto, 2 estudios aleatorizados que comparan más de 3 años de tratamiento adyuvante con IMB con el tratamiento estándar ( 3 años), se lanzaron en Europa y se encuentran en curso (28).

Joensuu, quien trató con éxito la primera paciente con TEGI metastásico en el año 2000, comentó que en este momento: "la duración estándar del IMB adyuvante es 3 años" (33). Tratamientos más prolongados no han probado ser beneficiosos, y no hay información de estudios clínicos mayores de 3 años. Por esto, se recomienda realizar imágenes de forma frecuente después de suspender el medicamento para detectar la recurrencia de forma temprana, recordar que la recurrencia del TEGI generalmente responde bien con el reinicio del IMB y que el riesgo de resistencia secundaria es muy bajo (30).

Actualmente, es importante recordar que una de las causas de recurrencia es una segunda mutación, en cuyo caso la recomendación es aumentar la dosis de IMB o pasar a una segunda línea de tratamiento; pero también se debe tener en consideración que se está hablando de terapia adyuvante y cuánto tiempo; es decir, luego de una cirugía completa (R0), con lesión de alto riesgo para recaída, se decide tratamiento adyuvante por un determinado tiempo. Al suspender el medicamento, el paciente presenta recurrencia de su enfermedad 6 a 9 meses después, que responde muy bien con la reiniciación del medicamento. La pregunta es: ¿se debería mantener el IMB?

Existen registros de pacientes con alto riesgo de recurrencia, que progresan después de suspender la administración del IMB luego de 3 años. Continuar o no el medicamento es una decisión complicada, por lo que se deben valorar variables individuales que pudieran medir el beneficio para ayudar a pacientes y médicos con una decisión más informada acerca de continuar el tratamiento (30).

No se informa de estudios clínicos con más de 5 años de tratamiento con IMB. La mayoría de los TEGI recurren después de interrumpir el tratamiento. El IMB se debe administrar de forma continua hasta que la enfermedad progrese, ya que la interrupción del tratamiento se traduce en progresión dentro del primer año (34). Para otros autores, la terapia adyuvante por mayor tiempo se justifica si se tiene la certeza que el riesgo de recurrencia es mayor del 50\% (30).

Los 2 primeros pacientes informados, luego de la cirugía sin enfermedad residual, recibieron 3 años de manejo con IMB y, después, cuando se suspendió el medicamento, consultaron por recaídas de su enfermedad. Actualmente, reciben IMB y muestran respuestas clínicas parciales iSe debe continuar con el medicamento de forma indefinida? Si la respuesta es sí, el tercer paciente sin evidencia actual de recaída no debería suspender el IMB.

De acuerdo conlo encontrado, la inhibición farmacológica del c-KIT es insuficiente para inducir apoptosis y erradicación de las células madres mutantes. Hallazgos clínicos y experimentales soportan la necesidad del tratamiento continuo con inhibidores de tirosina quinasa (ITK). La interrupción del tratamiento se asocia con una rápida repoblación de células cancerosas diferenciadas de una población intacta de células madres y progenitoras (35).

Se piensa que mientras se recibe imatinib, las células madre y progenitoras están dormidas y una vez se suspende el medicamento las células se despiertan. IMB se une a los sitios de activación del adenosín trifosfato (ATP), bloqueando la fosforilación y la activación de la cascada intracelular. Una vez se suspende el medicamento, su acción desaparece, por lo que los pacientes pueden responder durante años, pero por lo general este tratamiento no cura completamente la enfermedad (figura 1) (36).

$\mathrm{Si}$ se tuviera evidencia de que la terapia con IMB adyuvante cura a los pacientes con TEGI, un período finito de terapia podría ser posible; sin embargo, los estudios demuestran que al suspender el IMB, las recurrencias rápidamente comienza a aparecer (30).

Estos agentes ITK pueden mejorar la duración del control de la enfermedad, pero no logran la curación. Se necesitan terapias que puedan erradicar las células madre iniciales para lograr la curación que elude los tratamientos actuales con los ITK, como el IMB (35).

El advenimiento de la terapia con IMB para el TEGI metastásico mejoró el pronóstico de forma dramática. Por esto, si existe beneficio clínico se recomienda el tratamiento de forma indefinida porque la interrupción se relaciona con recaída o progresión de la enfermedad $(37,38)$.

\section{CONCLUSIONES}

Se sabe que la duración óptima de la terapia adyuvante con IMB en los pacientes con TEGI todavía no se conoce. Existen algunos grupos de pacientes que se pueden beneficiar de la terapia de por vida. 
La terapia adyuvante prolongada no incrementa significativamente los efectos adversos relacionados con el tratamiento.

El tratamiento adyuvante con IMB demostró reducción significativa en el riesgo de recurrencia; además, el IMB es el medicamento de primera línea adyuvante en el tratamiento del TEGI.

La sobrevida libre de recurrencia se correlaciona con la duración del tratamiento adyuvante. Los criterios de estratificación del riesgo deben establecer el paciente con alto riesgo de recurrencia que se va a beneficiar del tratamiento adyuvante y probablemente será también el que se favorezca de mayor tiempo con el medicamento.

En pacientes con riesgo de recurrencia establecido mayor del 50\%, se pudiera considerar un período más largo de 3 años de tratamiento adyuvante con IMB, sin que existan estudios con evidencia clínica suficiente que soporten esta recomendación. El estado mutacional del KIT tiene un impacto significativo en la respuesta al tratamiento. El IMB puede mejorar el control de la enfermedad pero no la curación.

\section{REFERENCIAS}

1. Corless C L. Gastrointestinal stromal tumors: what do we know now? Modern Pathology. 2014;27:S1-S16.

2. Ogata K, Mochiki E, Ojima H, et al. A Multicenter long term study of Imatinib treatment for japanese patients with unresectable or recurrent gastrointestinal stromal tumors. J Surg Oncol. 2014;110:942-6.

3. Hirota S, Isozaki K, Moriyama Y, et al. Gain of function mutation of c-KIT in human gastrointestinal stromal tumors. Science. 1998;279:577-80.

4. Cioffi A, Maki RG. GI stromal tumors: 15 years of lessons from a rare cancer. J Clin Oncol. 2015;33:1849-54.

5. Zhong JH, Ma L, Li LQ, et al. Adjuvant Imatinib for gastrointestinal stromal tumors: the current situation and problems. Scand J Of Gastroent. 2011;46:645-51.

6. Miettinen M, Makhlouf H, Sobin LH, et al. Gastrointestinal stromal tumors of the jejunum and ileum: a clinicopathologic, inmunohistochemical and molecular genetic study of 906 cases before imatinib with long term follow up. Am J Surg Pathol. Abril 2006;30(4):477-89.

7. Caram M, Schuetze S. Advanced or metastasic gastrointestinal stromal tumors: systemic treatment options. J Surg Oncol. 2011;104:888-95.

8. Blackstein ME, Corless CL, Ballman KV, et al. Risk assessment for tumor recurrence after surgical resection of localized primary gastrointestinal stromal tumor (GIST): North American Intergroup phase III trial ACOSOG Z9001. Presented at the 2010 Gastrointestinal Cancers Symposium: 22-24de enero de 2010; Orlando, FL, EE. UU.

9. Joensuu H, Eriksson M, Sundby HK, et al. One vs. three years of adjuvant imatinib for operable gastrointestinal stromal tumor: a randomized trial. JAMA. Marzo 2012;307(12):1265-72.
10. Maki R, Blay JY, Demetri G, et al. Key issues in the clinical management of gastrointestinal stromal tumors: an expert discussion. The Oncologist. 2015;20:823-30.

11. Reichardt P, Joensuu H, Blay JY. News fronts in the adjuvant treatment of GIST. Cancer Chemother Pharmacol. 2013;72:715-723.

12. Demetri G, Titton RL, Ryan DP, et al. Case 32-2004: a 68 year old man with a large retroperitoneal mass. N Engl J Med. 2004;351(17)21:1779-87.

13. Blay JY, Casali PG, Dei Tos AP, et al. Management of gastrointestinal stromal tumour: current practices and visions for the future. Oncology. 2015;89:1-13.

14. Demetri G, Mehren M, Antonescu C, et al. NCCN Task Force Report: update on the management of patients with gastrointestinal stromal tumors. J Natl Compr Canc Netw. 2010;8(2):S1-41.

15. ESMO. European Sarcoma Network Working Group: Gastrointestinal stromal tumors. ESMO Clinical Practice Guidelines for diagnosis, treatment and follow-up. Ann Oncol. 2012;23(17):vii49-55.

16. Bischof DA, Dodson R, Jimenez MC, et al. Adherence to guideliness for adjuvant imatinib therapy for GIST: a multiinstitutional analysis. J Gastrointest Surg. 2015;19:1022-8.

17. Sicklick JK, Lopez N. Optimizing surgical and imatinib therapy for the treatment of gastrointestinal stromal tumors. J Gastroint Surg. 2013;17:1997-2006.

18. Miettinen M Lasota J. Gastrointestinal stromal tumors: pathology and prognosis at different sites. Semin Diagn Pathol. 2006;23(2):70-83.

19. Telzlaff E, Davey M. Optimizing adherence to adjuvant Imatinib in gastrointestinal stromal tumor. J Adv Pract Oncol. 2013;4(4):238-50.

20. Gold JS, Gonen M, Gutierrez A, et al. Development and validation of a prognostic nomogram for recurrence free survival after complete surgical resection of localized primary gastrointestinal stromal tumor: a retrospective analysis. Lancet Oncol. 2009;10(11):1045-52.

21. Rossi S, Miceli R, Messerini L, et al. Natural history of imatinib naive GISTs: a retrospective analysis of 929 cases with long term follow and development of a survival nomogram base don mitotic index and size as continuous variables. Am J Surg Pathol. 2011;35:1646-56.

22. Pracucho EM, Lopes LR, Zanatto RM, et al. Profile of patients with gastrointestinal stromal tumors. Arq Bras Cir Dig. 2015;28(2):124-7.

23. Trent JC, Subramanian MP. Managing GIST in the Imatinib era: optimization of adjuvant therapy. Expert Rev Anticancer Ther. 2014;14(12):1445-59.

24. Dematteo RP, Ballman KV, Antonescu CR, et al. Long term results of adjuvant imatinib mesylate in localized high-risk primary gastrointestinal stromal tumor. ACOSOG Z9000 Intergroup phase 2 Trial. Ann Surg. 2013;258(3):422-9.

25. Dematteo RP, Ballman KV, Antonescu CR, et al. Adjuvant Imatinib mesylate after resection of localized primary gastrointestinal stromal tumours: a randomized, double-blind placebo controlled trial. Lancet. 2009:373(9669):1097-104. 
26. Niazi AK, Kaley K, Saif W. Gastrointestinal stromal tumor of colon: a case report and review of literature. Anticancer Research. 2014;34:2547-50.

27. Le Cesne A, Coquard IR, Bui BN, et al. Discontinuation of Imatinib in patients with advanced gastrointestinal stromal tumors after 3 years of treatment: an open label multicenter randomized phase 3 trial. Lancet Oncol. 2010;11:942-9.

28. Novartis Pharmaceuticals. Five year adjuvant Imatinib mesylate (Gleevec) in gastrointestinal stromal tumor (GIST). 2012. Disponible en: http//clinicaltrials.gov/ct2/show/ NCT00867113

29. Ray Coquard IL, Bin Bui N, Adenis A, et al. Risk of relapse with Imatininb (IM) discontinuation at 5 years in advanced GIST patients: results of the prospective BFR14 randomized phase III study comparing interruption versus continuation of IM at 5 years of treatment: a French Sarcoma Group Study. J Clin Oncol (Meeting Abstracts). 2010;28:10032.

30. Knox P. Adjuvant Imatinib study updates results. 15 de diciembre de 2015. Disponible en: https://liferaftgroup. org/2015/12/adjuvant-imatinib-study-updates-results/

31. Le Cesne A, Blay J Y, Reichardt P, et al. Optimizing tyrosine kinase inhibitor therapy in gastrointestinal stromal tumors: exploring the benefits of continuous kinase suppression. The Oncolgyst. 2013;18:1192-9.

32. Reichardt P, Blay JY, Boukovinas I, et al. Adjuvant therapy in primary GIST: state of the art. Ann of Oncol.2012;23:2776-81.

33. Joensuu H, Roberts PJ, Sarlomo Rikala M, et al. Effect of the tyrosine kinase inhibitor ST1571 in a patient with a metastasic gastrointestinal stromal tumor. N Engl J Med. 2001;344:1052-6.

34. Joenssu H, Hohenberger P, Corless CH. Gastrointestinal stromal tumor. Lancet. 2013;382:973-83.

35. Heinrich MC. Imatinib treatment of metastasic GIST: don't stop (believing). Lancet Oncol. 2010;11:910-1.

36. Bauer S, Fletcher J. El descubrimiento de nuevas terapias es posible si comprendemos como se desarrolla un GIST. Disponible en: http://alianzagist.org/assets/nuevas-terapias.pdf

37. Balachandran VP, DeMatteo RP. Targeted therapy for cancer. The Gastrointestinal Stromal Tumor Model. Surg Oncol Clin N Am. 2013;22:805-21.

38. Balachandran VP, DeMatteo RP. Gastrointestinal stromal tumors. Who should get imatinib and for how long? Advances in Surgery. 2014;48:165-83. 